\title{
ARTICLE \\ High-throughput screening campaign identifies a small molecule agonist of the relaxin family peptide receptor 4
}

\author{
Guang-yao Lin ${ }^{1,2,3}$, Lin Lin ${ }^{4}$, Xiao-qing Cai ${ }^{1}$, An-tao Dai ${ }^{1}$, Yue Zhu ${ }^{1,3}$, Jie Li ${ }^{1,3}$, Qing Liu ${ }^{1}$, De-hua Yang ${ }^{1,3}$, Ross A. D. Bathgate ${ }^{5}$ and \\ Ming-wei Wang ${ }^{1,2,3,4}$
}

Relaxin/insulin-like family peptide receptor 4 (RXFP4) is a class A G protein-coupled receptor (GPCR), and insulin-like peptide 5 (INSL5) is its endogenous ligand. Although the precise physiological role of INSL5/RXFP4 remains elusive, a number of studies have suggested it to be a potential therapeutic target for obesity and other metabolic disorders. Since selective agonists of RXFP4 are scarcely available and peptidic analogs of INSL5 are hard to make, we conducted a high-throughput screening campaign against 52,000 synthetic and natural compounds targeting RXFP4. Of the 109 initial hits discovered, only 3 compounds were confirmed in secondary screening, with JK0621-D008 displaying the best agonism at human RXFP4. Its Sconfiguration stereoisomer (JK1) was subsequently isolated and validated by a series of bioassays, demonstrating a consistent agonistic effect in cells overexpressing RXFP4. This scaffold may provide a valuable tool to further explore the biological functions of RXFP4.

Keywords: RXFP4; INSL5; high-throughput screening; agonist; JK1

Acta Pharmacologica Sinica (2020) 41:1328-1336; https://doi.org/10.1038/s41401-020-0390-x

\section{INTRODUCTION}

Relaxin/insulin-like family peptide receptor 4 (RXFP4), also known as GPCR142 or GPR100, is a class A G protein-coupled receptor (GPCR) [1-3]. It is primarily expressed in peripheral tissues with the highest expression in the colon [1]. Insulin-like peptide 5 (INSL5), a member of the relaxin family of peptides with two chains and three disulfide bonds [4, 5], is the endogenous ligand of RXFP4 [1]. The highest expression of INSL5 is in the colon of humans [1] and mice [6], with lower expression in other tissues in humans and mice, including the pituitary gland, testes, and kidneys. INSL5 activates RXFP4 through the pertussis toxin (PTX)-sensitive $\mathrm{Ga}_{\mathrm{i} / \mathrm{o}}$ signaling pathway, resulting in the inhibition of cyclic adenosine monophosphate (cAMP) accumulation [1, 7] and increases in phosphorylation of extracellular regulated protein kinases $1 / 2$ (ERK1/2) [7, 8], either in heterologous systems overexpressing RXFP4 or in cells expressing RXFP4 endogenously. In addition, RXFP4 also undergoes $\beta$-arrestin-mediated signaling following ligand stimulation [7].

Since the precise functions of INSL5/RXFP4 remain unclear, major efforts have been made to study the physiology of this pair. It has been reported that RXFP4-knockout mice showed a decreased serum glucose level, increased body weight and higher fat content, along with suppressed food intake and water consumption [9]. RXFP4 is thus involved in fat metabolism and appetite, and the treatment of obesity or anorexia may be achieved by upregulating RXFP4 expression or treatment with an RXFP4 agonist. In addition, INSL5-deficient mice also displayed impairment in glucose homeostasis and fertility [10]. A recent study found that INSL5 is one of the products of colonic enteroendocrine L-cells, which also secrete glucagon-like peptide-1 (GLP-1), oxyntomodulin and peptide YY (PYY). In fact, INSL5 has been identified as the second orexigenic gastrointestinal hormone after ghrelin [11]. Our previous work established the insulinotrophic action of INSL5 in vitro and in vivo [8]. Altogether, these results make the INSL5/ RXFP4 system a potential therapeutic target for obesity and other metabolic disorders.

A recent study [12] identified an indole-containing scaffold as a dual agonist for RXFP3 and RXFP4. However, no structural modification around this scaffold was disclosed for further exploration. As selective agonists of RXFP4 are scarcely available and peptidic analogs of INSL5 are hard to make [13, 14], we developed and validated a CAMP measurement-based highthroughput screening (HTS) assay targeting RXFP4. It was employed in a large-scale random screening campaign of 52,000 synthetic and natural compounds aiming to discover small molecule modulators of this receptor.

\section{MATERIALS AND METHODS}

Reagents

Human INSL5 (hINSL5), mouse INSL5 (mINSL5), relaxin-3 and R3/I5 were purchased from Phoenix Pharmaceuticals (Burlingame, CA, USA). Their HPLC and mass spectrometry data are shown in

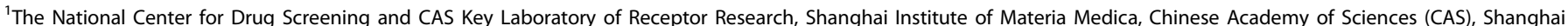



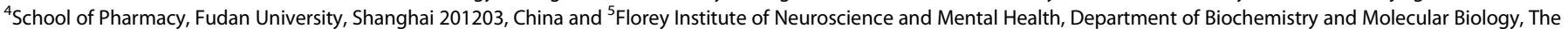
University of Melbourne, Parkville, VIC 3052, Australia

Correspondence: De-hua Yang (dhyang@simm.ac.cn) or Ross A. D. Bathgate (bathgate@florey.edu.au) or Ming-wei Wang (mwwang@simm.ac.cn)

Received: 18 December 2019 Accepted: 21 February 2020

Published online: 31 March 2020 
a

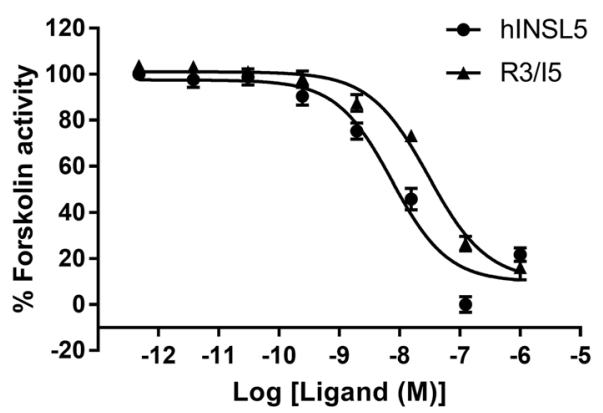

b

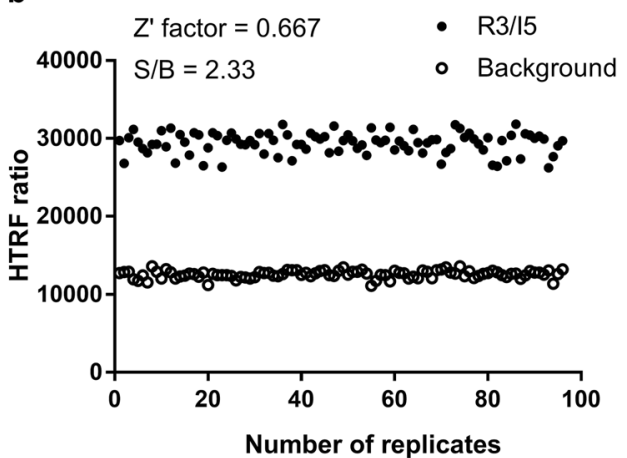

Fig. 1 Validation of HTS assay. a Dose-response characteristics of hINSL5 and R3/I5 in hRXFP4-CHO cells under the optimized assay conditions. b Z' factor of HTS assay. A total of 160 replicates of R3/15 and background signals were studied

Supplementary Fig. S2. All of the small molecules were from an inhouse chemical compound library (https://www.cncl.org.cn) unless otherwise indicated. JK1 was discovered and resynthesized in our laboratory, and its ${ }^{1} \mathrm{H}$ NMR is provided in Supplementary Fig. S3. Compound $\mathbf{4}$ was synthesized by SYNthesis (Shanghai, China). LANCE Ultra CAMP and AlphaScreen SureFire pERK1/2 assay kits were obtained from PerkinElmer (Waltham, MA, USA). Forskolin, 3-isobutyl-1-methylxanthine (IBMX), 2-(2-amino-3methoxyphenyl)-4H-1-benzopyran-4-one (PD98059), dimethyl sulfoxide (DMSO) and bovine serum albumin (BSA) were supplied by Sigma-Aldrich (St. Louis, MO, USA). Dulbecco's modified Eagle's medium (DMEM), Ham's F-12 nutrient mixture (F12), Dulbecco's modified Eagle's medium/nutrient mixture F-12 (DMEM/ F12), Hank's balanced salt solution (HBSS), L-glutamine, penicillin-streptomycin, $\quad 0.25 \%$ trypsin-EDTA, Dulbecco's phosphate-buffered saline (DPBS), fetal bovine serum (FBS), PTX, hygromycin $B$, geneticin and Fluo-4-AM were all purchased from Life Technologies (Carlsbad, CA, USA). Coelenterazine-h was procured from YEASEN Biotechnology (Shanghai, China), and the assay plates were from Greiner (Frickenhausen, Germany), Corning (Tewksbury, MA, USA) and PerkinElmer.

\section{Constructs}

The pEF5/FRT/V5-DEST destination vector and its modified vector with Rluc8 in the frame of the gateway cassette followed by an internal ribosome entry site and either $\beta$-arrestin- 1 or $\beta$ arrestin-2 Venus fusion (pEF5/FRT-Rluc8-ArrVen) were kindly provided by Dr. Patrick M. Sexton (Monash University, Parkville, Victoria, Australia). Human RXFP3 (NCBI Reference Sequence: NM_016568.3), human (NCBI Reference Sequence: NM_181885.3), and mouse (NCBI Reference Sequence: NM_181817.1) RXFP4 receptor sequences were amplified from the original pCMV6 construct (OriGene Technologies, Rockville, MD, USA) and subcloned into pEF5/FRT/V5-DEST and pEF5/FRTRluc8-ArrVen destination vectors using Gateway Technology (Invitrogen, Carlsbad, CA, USA). All constructs were confirmed by DNA sequencing.

\section{Cell culture}

The above constructs were transfected into Chinese hamster ovary (Flpln-CHO) cells via the Flpln system (Invitrogen), and hygromycin B was used for polyclonal selection. The cells stably expressing human RXFP4 or mouse RXFP4 (mRXFP4-FIplnCHO) were maintained in F12 supplemented with $10 \%$ (v/v) FBS and $800 \mu \mathrm{g} / \mathrm{mL}$ hygromycin $\mathrm{B}$ at $37^{\circ} \mathrm{C}$ in $5 \% \mathrm{CO}_{2}$. CHO-K1 cells stably expressing human RXFP4 (hRXFP4-CHO) [15] or RXFP3 (hRXFP3$\mathrm{CHO}$ ) [16] were maintained in DMEM/F12 supplemented with $10 \%(\mathrm{v} / \mathrm{v}) \mathrm{FBS}, 2 \mathrm{mM}$ L-glutamine, 100 units $/ \mathrm{mL}$ penicillin and
$100 \mu \mathrm{g} / \mathrm{mL}$ streptomycin at $37^{\circ} \mathrm{C}$ in $5 \% \mathrm{CO}_{2} . \mathrm{Ga}_{16}$ - $\mathrm{CHO}$ cells (RDHGA16; Molecular Devices, Sunnyvale, CA, USA) were maintained in $\mathrm{F} 12$ containing $10 \%(\mathrm{v} / \mathrm{v}) \mathrm{FBS}, 2 \mathrm{mM}$ L-glutamine, 100 units $/ \mathrm{mL}$ penicillin, $100 \mu \mathrm{g} / \mathrm{mL}$ streptomycin, and $200 \mu \mathrm{g} / \mathrm{mL}$ hygromycin $\mathrm{B}$ at $37^{\circ} \mathrm{C}$ in $5 \% \mathrm{CO}_{2}$. $\mathrm{CHO}$ cells stably expressing human RXFP3 or RXFP4 and $\mathrm{Ga}_{16}$ were generated by transfection of the hRXFP3-pCMV6 or hRXFP4-pCMV6 construct into $\mathrm{Ga}_{16}-\mathrm{CHO}$ cells (hRXFP3- $\mathrm{Ga}_{16}-\mathrm{CHO}$ and hRXFP4-Ga ${ }_{16}-\mathrm{CHO}$, respectively), and geneticin $(600 \mu \mathrm{g} / \mathrm{mL})$ was used for selection. They were maintained in F12 containing $10 \%(\mathrm{v} / \mathrm{v}) \mathrm{FBS}, 2 \mathrm{mM}$ L-glutamine, and $600 \mu \mathrm{g} / \mathrm{mL}$ geneticin at $37^{\circ} \mathrm{C}$ in $5 \% \mathrm{CO}_{2}$. Human embryonic kidney 293 cells containing SV40 large Tantigen (HEK293T) were cultured in DMEM supplemented with $10 \%(\mathrm{v} / \mathrm{v}) \mathrm{FBS}, 2 \mathrm{mM}$ L-glutamine, 100 units $/ \mathrm{mL}$ penicillin and $100 \mu \mathrm{g} / \mathrm{mL}$ streptomycin at $37^{\circ} \mathrm{C}$ in $5 \% \mathrm{CO}_{2}$.

\section{Compound library}

A collection of 52,000 synthetic and natural compounds stored at the Chinese National Compound Library (https://www.cncl.org.cn) was used in this study. The structural diversity covers heterocycles, lactams, sulfonates, sulfonamides, amines, secondary amides, etc. All of the compounds are highly pure, and the stock, presolubilized in $100 \%$ DMSO solution, was applied to the primary screening.

\section{HTS campaign}

The HTS assay, developed to measure the inhibition of forskolinstimulated CAMP production in hRXFP4-CHO cells, was carried out in white 384-well plates (PerkinElmer). Briefly, 64 wells of the outer four columns on both sides of a plate were used for the positive control ( $500 \mathrm{nM} \mathrm{R3/15}$ in $0.5 \%$ DMSO) and negative control (0.5\% DMSO), each with 32 replicates. The rest of the wells in the center columns were loaded with individual compounds to be screened with an average final concentration of $10 \mu \mathrm{M}$ in a single-dose format without replicates. Timeresolved fluorescence resonance energy transfer (TR-FRET) signals were assessed by an EnVision multilabel plate reader (PerkinElmer). The $Z^{\prime}$ factor, coefficient of variation (CV) and signal-to-background ratio (S/B) were evaluated according to the literature [17]. The positive control was regarded as $100 \%$ activation, while the negative control represented no activation. Compounds showing greater than $50 \%$ activation were considered hits, and secondary screening using the same assay was conducted to eliminate false positives.

cAMP accumulation assay

For off-target examination, inhibition of forskolin-induced cAMP accumulation by compounds was carried out in parental $\mathrm{CHO}$ 

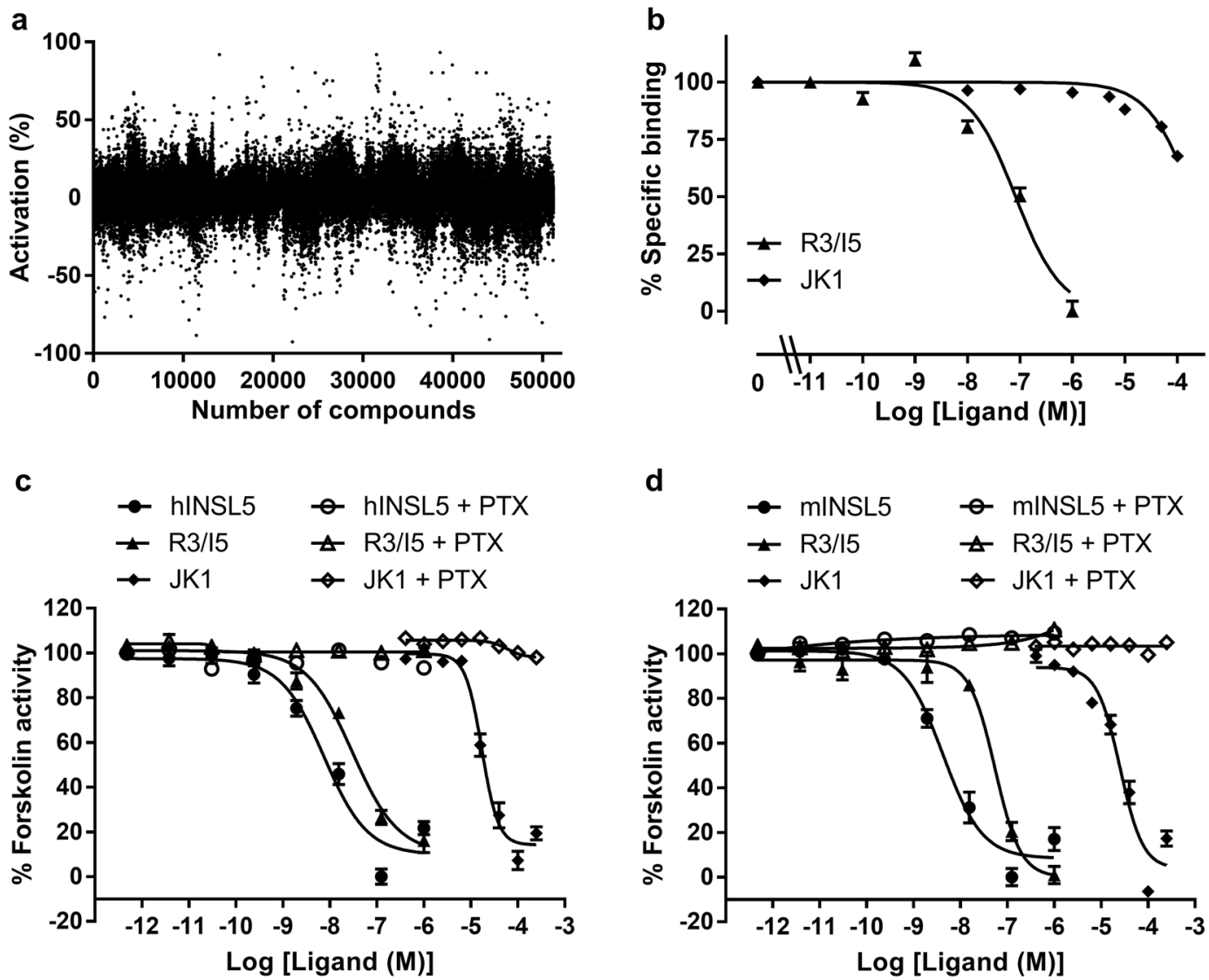

Fig. 2 HTS campaign to discover potential RXFP4 agonists. a Large-scale screening of 52,000 synthetic and natural compounds. $\mathbf{b}$ Competitive binding of JK1 to RXFP4. c, d Activation of human (left panel) and mouse (right panel) RXFP4 by JK1 and this agonistic effect could be abolished when the cells were treated with PTX $(100 \mathrm{ng} / \mathrm{mL}, 18 \mathrm{~h})$ before ligand stimulation

Table 1. Dose-response characteristics and $\mathrm{pEC}_{50}$ values of three confirmed hits in the CAMP assay

\begin{tabular}{|c|c|c|c|c|}
\hline Compound & Structure & Chemical formula & Molecular weight & $\mathrm{pEC}_{50}$ \\
\hline JK0621-D008 & & $\mathrm{C}_{21} \mathrm{H}_{17} \mathrm{ClN}_{4} \mathrm{O}_{2}$ & 392.84 & $5.3 \pm 0.14$ \\
\hline JK0644-G008 & & $\mathrm{C}_{13} \mathrm{H}_{12} \mathrm{~F}_{3} \mathrm{~N}_{3} \mathrm{OS}$ & 315.31 & $4.5 \pm 0.39$ \\
\hline RUS0868-F010 & & $\mathrm{C}_{17} \mathrm{H}_{18} \mathrm{~N}_{4} \mathrm{O}_{2}$ & 310.36 & $4.9 \pm 0.89$ \\
\hline
\end{tabular}


cells. For selective agonistic effect validation, compounds were tested for their ability to inhibit cAMP activity in hRXFP3-CHO, hRXFP4-CHO, and mRXFP4-FIplnCHO. Cells were seeded at a density of $8 \times 10^{5}$ cells $/ \mathrm{mL}$ and stimulated with different concentrations of compounds plus $500 \mathrm{nM}$ forskolin for $40 \mathrm{~min}$ at room temperature (RT) in the presence of $500 \mu \mathrm{M}$ IBMX. The Eu-cAMP tracer and ULight-anti-cAMP working solution were then added followed by a $60-\mathrm{min}$ incubation at RT. TR-FRET signals were detected by EnVision with excitation at 320 or $340 \mathrm{~nm}$ and emission at $665 \mathrm{~nm}$. Data were normalized to the maximal response of hINSL5 or mINSL5.

\section{pCRE reporter gene assay}

JK1 was tested for its ability to inhibit forskolin-induced pCRE (cAMP-response element) reporter gene activity in CHO-RXFP3, CHO-RXFP4, or parental CHO cells transfected with a pCRE $\beta$ galactosidase reporter plasmid as previously described [15]. Cells were stimulated with $1 \mu \mathrm{M}$ forskolin plus or minus increasing concentrations of INSL5, R3/15, compound $\mathbf{4}$ or JK1 for 6 h. Each compound was tested in triplicate, and each experiment was performed independently at least three times. Data are expressed as the \% forskolin activity and analyzed and plotted using Prism software version 8 (GraphPad, San Diego, CA, USA) as the means \pm SEM of the pooled data.

\section{Binding assay}

Eu-mINSL5 competition binding assays were performed in $\mathrm{CHO}$ RXFP4 cells as previously described [15]. The synthesis and characterization of the Eu-mINSL5 ligand was described previously [15]. Time-resolved fluorescent measurements were carried out at an excitation wavelength of $340 \mathrm{~nm}$ and an emission wavelength of $614 \mathrm{~nm}$ on a BMG POLARstar plate reader (BMG Labtech, Melbourne, Australia). Pooled data are presented as the means \pm SEM of specific binding and were fitted using a one-site binding curve in Prism software (GraphPad).

ERK1/2 phosphorylation assay

The ERK1/2 phosphorylation assay was performed in hRXFP3-CHO and hRXFP4-CHO cells $(40,000$ cells/well) and incubated overnight with serum containing DMEM/F12 at $37^{\circ} \mathrm{C}$ in $5 \% \mathrm{CO}_{2}$. A timecourse study was carried out to determine the optimal stimulation window. The cells were washed twice with DPBS and serum starved in serum-free DMEM/F12 for $6 \mathrm{~h}$ followed by stimulation with different concentrations of compounds or $10 \%$ FBS for 6 min or $7 \mathrm{~min}$, respectively. After incubation, the cells were lysed at RT with shaking $(\sim 350 \mathrm{rpm})$ for $10 \mathrm{~min}$, and then $5 \mu \mathrm{L}$ of the lysate together with $8.5 \mu \mathrm{L}$ of the reaction mix containing both donor and acceptor beads was transferred to a white plate followed by 2 min of gentle agitation and $1 \mathrm{~h}$ of incubation at $37^{\circ} \mathrm{C}$. AlphaScreen signals (counts) were measured on an EnVision with excitation at $680 \mathrm{~nm}$ and emission at 520-620 nm. Data were normalized to the response of $10 \%$ FBS.

\section{Calcium mobilization assay}

hRXFP3-Ga $-\mathrm{Ga}_{16}-\mathrm{CHO}$, hRXFP4-Ga ${ }_{16}-\mathrm{CHO}$, and $\mathrm{Ga}_{16}-\mathrm{CHO}$ cells were seeded into 96-well plates at a density of 30,000 cells/well and incubated for $24 \mathrm{~h}$ at $37^{\circ} \mathrm{C}$ in $5 \% \mathrm{CO}_{2}$. They were washed twice with HBSS and reacted with $2 \mu \mathrm{M}$ Fluo-4-AM in HBSS supplemented with $2.5 \mathrm{mM}$ probenecid for $1 \mathrm{~h}$ at $37{ }^{\circ} \mathrm{C}$ in $5 \% \mathrm{CO}_{2}$. Different concentrations of compounds were added, followed by analysis of the intracellular calcium mobilization on an FLIPR instrument (Molecular Devices) with excitation at 470-495 $\mathrm{nm}$ and emission at $515-575 \mathrm{~nm}$. Data were normalized to the baseline response of each compound.

\section{$\beta$-Arrestin recruitment assay}

Flpln-CHO cells stably expressing hRXFP3-Rluc8 or hRXFP4-Rluc8 and either $\beta$-arrestin-1-Venus or $\beta$-arrestin-2-Venus were seeded


Fig. 3 Specificity of JK1 for RXFP4. a-c JK1 was not active in hRXFP3$\mathrm{CHO}, 5-\mathrm{HT}_{1 \mathrm{~B}}$ receptor- $\mathrm{CHO}$ and $\mathrm{CHO}$ cells, respectively

into white plates at a density of 30,000 cells/well and incubated for $24 \mathrm{~h}$ at $37^{\circ} \mathrm{C}$ in $5 \% \mathrm{CO}_{2}$. Cells were rinsed once with HBSS and incubated with fresh HBSS for $30 \mathrm{~min}$ at $37^{\circ} \mathrm{C}$ in $5 \% \mathrm{CO}_{2}$ thereafter. Then, they were loaded with $5 \mu \mathrm{M}$ coelenterazine-h in HBSS and incubated for $5 \mathrm{~min}$. Compounds with different concentrations were added, and bioluminescence resonance energy transfer (BRET) signals were detected on an EnVision with dual emission at $470 \mathrm{~nm}$ and $535 \mathrm{~nm}$ before and after the addition of ligands. The BRET values were calculated by subtracting the ratio of emission at $535 \mathrm{~nm}$ over emission at $470 \mathrm{~nm}$ for the vehicle-treated cell samples from the same ratio obtained in the compound-treated cell samples. 


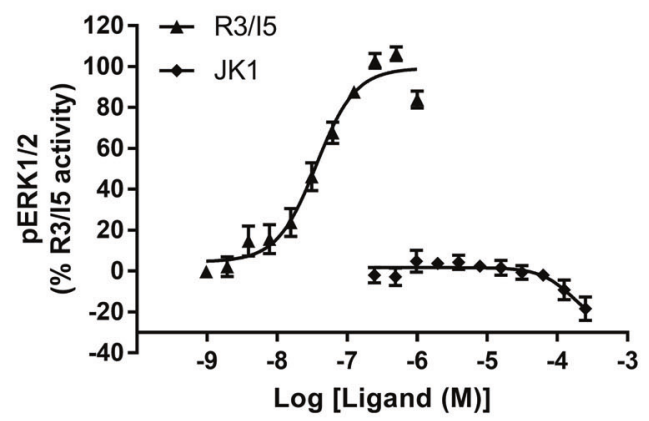

C

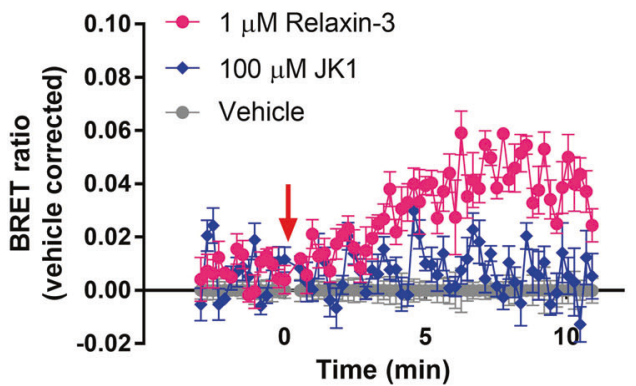

b



d

RXFP3 interaction with $\beta$-arrestin 2

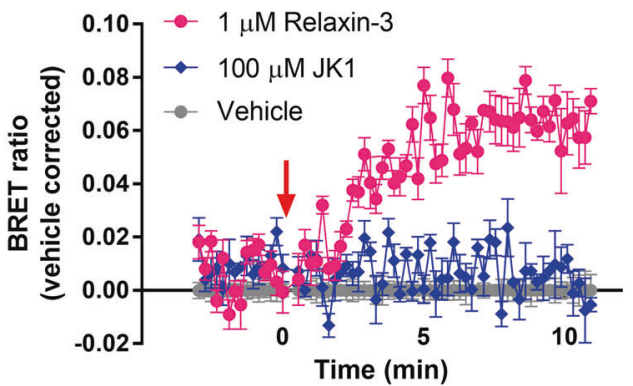

Fig. 4 Specificity of JK1 for RXFP3. a JK1 had no effect on RXFP3-mediated ERK1/2 phosphorylation in hRXFP3-CHO cells. b JK1 displayed no agonistic activity in hRXFP3-G $\alpha_{16}-\mathrm{CHO}$. c, d JK1 did not promote RXFP3 interaction with $\beta$-arrestin $1 / 2$

Cytotoxicity assay

Cytotoxicity was assessed in parental CHO and HEK293T cells using the Cell Counting Kit-8 (CCK-8; Dojindo, Kumamoto, Japan). Briefly, cells were seeded into 96-well plates at a density of 30,000 cells/well and incubated overnight. The next day, different concentrations of compounds were added followed by $24 \mathrm{~h}$ of incubation, and CCK-8 solution was then loaded and incubated for $1 \mathrm{~h}$. Absorbance values at $450 \mathrm{~nm}$ were quantitated on a SpectraMax M5 plate reader (Molecular Devices). Data were normalized to the vehicle-treated samples.

Statistical analysis

Dose-response data were analyzed with Prism software (GraphPad) using a sigmoidal model with variable slope. Data were expressed as the means \pm SEM of at least three independent experiments. Statistical significance was determined using twotailed Student's $t$ tests, and $P<0.05$ was considered statistically significant.

\section{RESULTS}

Assay validation

Forskolin dose-response curves were generated at different cell densities and employed to determine the optimal cell density for cAMP assay development. Then, 4000 cells per well and $500 \mathrm{nM}$ forskolin were selected for the HTS campaign. Under the optimized conditions, the $\mathrm{pEC}_{50}$ values of hINSL5 and R3/15 were $8.12 \pm 0.13$ and $7.52 \pm 0.10$, respectively (Fig. 1a), consistent with that reported in the literature [1].

Assay performance

In the CAMP accumulation assay, signals evoked by positive (R3/15) and negative (background) controls were studied. As shown in Fig. $1 \mathrm{~b}$, the CV value was $4.67 \%$ for $\mathrm{R} 3 / 15$ and $3.92 \%$ for the background. The $Z^{\prime}$ factor calculated was 0.667 with an S/B ratio of
2.33. These characteristics indicate that the assay system is of high quality and well suited for HTS [17].

Lead identification

In an attempt to discover selective nonpeptidic agonists for RXFP4, we screened 52,000 synthetic and natural compounds against $\mathrm{CHO}-\mathrm{K} 1$ cells stably expressing the human receptor. As shown in Fig. 2a, primary screening yielded 109 initial hits showing greater than $50 \%$ activation. However, most of them failed in the secondary screening or dose-response studies, and only three hits were confirmed (Table 1) in the CAMP assay. Among them, JK0621-D008 displayed the best activity on hRXFP4$\mathrm{CHO}$. Since it is a racemic mixture, chiral resolution and single crystal X-ray diffraction led to the identification of the $S$ configuration stereoisomer JK1 (data not shown), which showed $\mathrm{pEC}_{50}$ values of $4.76 \pm 0.04$ for human RXFP4 and $4.60 \pm 0.07$ for mouse RXFP4 in the CAMP accumulation assay (Fig. 2c, d). Importantly, the actions of JK1 and control peptides were abolished by the $\mathrm{Ga}_{\mathrm{i} / \mathrm{o}}$ inhibitor PTX. In addition, JK1 was capable of competing with Eu-labeled INSL5 binding to human RXFP4 with an affinity $\left(\mathrm{p} K_{\mathrm{i}}=4.48 \pm 0.09, n=3\right)$ that matched its activity (Fig. 2b).

The specificity of JK1 for RXFP4 was not only tested in CHO-K1 cells stably expressing human relaxin/insulin-like family peptide receptor 3 (RXFP3), which is the most closely related receptor to RXFP4 [18], but also evaluated in CHO cells overexpressing the human $5-\mathrm{HT}_{1 \mathrm{~B}}$ receptor, which also couples to $\mathrm{Ga}_{\mathrm{i}}$ or $\mathrm{Ga}_{\mathrm{o}}$. Similar to hINSL5, JK1 did not activate these cells, whereas R3/l5 and dihydroergotamine (DHE) each dose-dependently stimulated their cognate receptors (Fig. 3a-c). A comparative activity study on RXFP3 with JK1 showed that JK1 did not induce RXFP3-mediated ERK1/2 phosphorylation, whereas R3/15 stimulated RXFP3 in a dose-dependent manner (Fig. 4a). Although relaxin-3 displayed an agonistic effect on RXFP3 in the calcium mobilization assay, JK1 showed no activation in CHO cells coexpressing RXFP3 and 
$\mathrm{Ga}_{16}$ (Fig. 4b). Moreover, relaxin-3 $(1 \mu \mathrm{M})$ time-dependently promoted the interaction between RXFP3 and $\beta$-arrestin $1 / 2$, which was not observed for JK1 (Fig. 4c, d). These results thus suggest that JK1 has no effect on the RXFP3-mediated signaling pathways.

In vitro characterization

The activity of JK1 was confirmed in a well-characterized pCRE reporter gene assay. JK1 demonstrated full agonistic activity at RXFP4 in these assays with more potent effects than in the direct cAMP assay $\left(\mathrm{pEC} 50=5.58 \pm 0.05\right.$; hINSL5 $\mathrm{pEC}_{50}=8.62 \pm$ 0.05) (Fig. 5a). JK1 showed very limited activity at the related RXFP3 receptor, while the recently reported RXFP4 agonist compound 4 demonstrated potent cross-reactivity (Fig. 5b). Importantly, JK1 demonstrated no activity in parental $\mathrm{CHO}$ cells (Fig. 5c).

It is well recognized that activation of $\mathrm{Ga}_{\mathrm{i} / \mathrm{o}}$-coupled receptors generally mediates ERK $1 / 2$ phosphorylation via $\beta \gamma$ subunits [19-21], and INSL5 has been demonstrated to activate ERK1/2 phosphorylation in CHO-RXFP4 cells [7]. JK1 was therefore assessed in the ERK1/2 phosphorylation assay for potency analysis. As shown in Fig. 6a, JK1 activated ERK1/2 phosphorylation in a dose-dependent manner ( $\mathrm{pEC} 50=4.85 \pm 0.07$ ), as did hINSL5 and $\mathrm{R} 3 / 15$. This effect was abolished by the $\mathrm{Ga}_{\mathrm{i} / \mathrm{o}}$ inhibitor PTX or downregulated by the MEK (mitogen-activated protein kinase kinase) inhibitor PD98059. These results indicate that JK1 activates the RXFP4-mediated ERK signaling pathway.

Because stimulation of RXFP4 results in calcium mobilization in the presence of the chimeric $\mathrm{G}$ protein $\mathrm{Ga}_{16}[1,8]$, JK1 was studied for its effect on intracellular calcium levels. In $\mathrm{CHO}$ cells coexpressing RXFP4 and $\mathrm{Ga}_{16}$, JK1 elicited dose-dependent calcium mobilization with a $\mathrm{pEC}_{50}$ value of $4.87 \pm 0.05$. This phenomenon was not observed in $\mathrm{CHO}$ cells expressing $\mathrm{Ga}_{16}$ alone, implying that the action requires the presence of RXFP4 (Fig. 6b).

Similar to many other GPCRs, RXFP4 also undergoes $\beta$-arrestinmediated signaling upon ligand stimulation [7]. Therefore, we examined the effect of JK1 on $\beta$-arrestin recruitment. As shown in Fig. $6 \mathrm{c}, \mathrm{R} 3 / \mathrm{I5}(1 \mu \mathrm{M})$ strongly and time-dependently promoted the interaction between RXFP4 and $\beta$-arrestin $1 / 2$, and treatment with hINSL5 $(1 \mu \mathrm{M})$ or JK1 $(100 \mu \mathrm{M})$ also induced $\beta$-arrestin $1 / 2$ recruitment (albeit weakly), reflecting the lower potency of the ligands.

The cytotoxicity of JK1 in HEK293T and CHO cells was assessed, which demonstrated that compared with hINSL5, JK1 exhibited a certain degree of toxicity at high concentrations: $\mathrm{plC}_{50}=4.36 \pm$ 0.05 for HEK293T cells and $\mathrm{plC}_{50}=3.74 \pm 0.04$ for $\mathrm{CHO}$ cells (Supplementary Fig. S1).

\section{DISCUSSION}

The relaxin family peptide receptors (RXFPs) and their cognate ligands play important roles in diverse physiological processes [22], including activities in the central nervous, cardiovascular, and reproductive systems as well as metabolic control. Among them, RXFP4 and its endogenous ligand INSL5 have been linked to insulin secretion and appetite $[8,11]$, thereby making them a potential therapeutic target for obesity and other metabolic disorders. Given the complexity of the interaction between RXFP4 and INSL5 [23], it is not surprising that the number of ligands selective for the receptor is rather limited. It is also well recognized that the synthesis of INSL5 and its peptide analogs is technically challenging $[13,14]$, and nonpeptidic agonists are thus in high demand. To date, although a handful of small molecule modulators exist for RXFP members [12, 24-26], including a series of small molecule dual agonists for RXFP3 and RXFP4 [12], nonpeptidic agonists selective for RXFP4 have not yet been discovered.
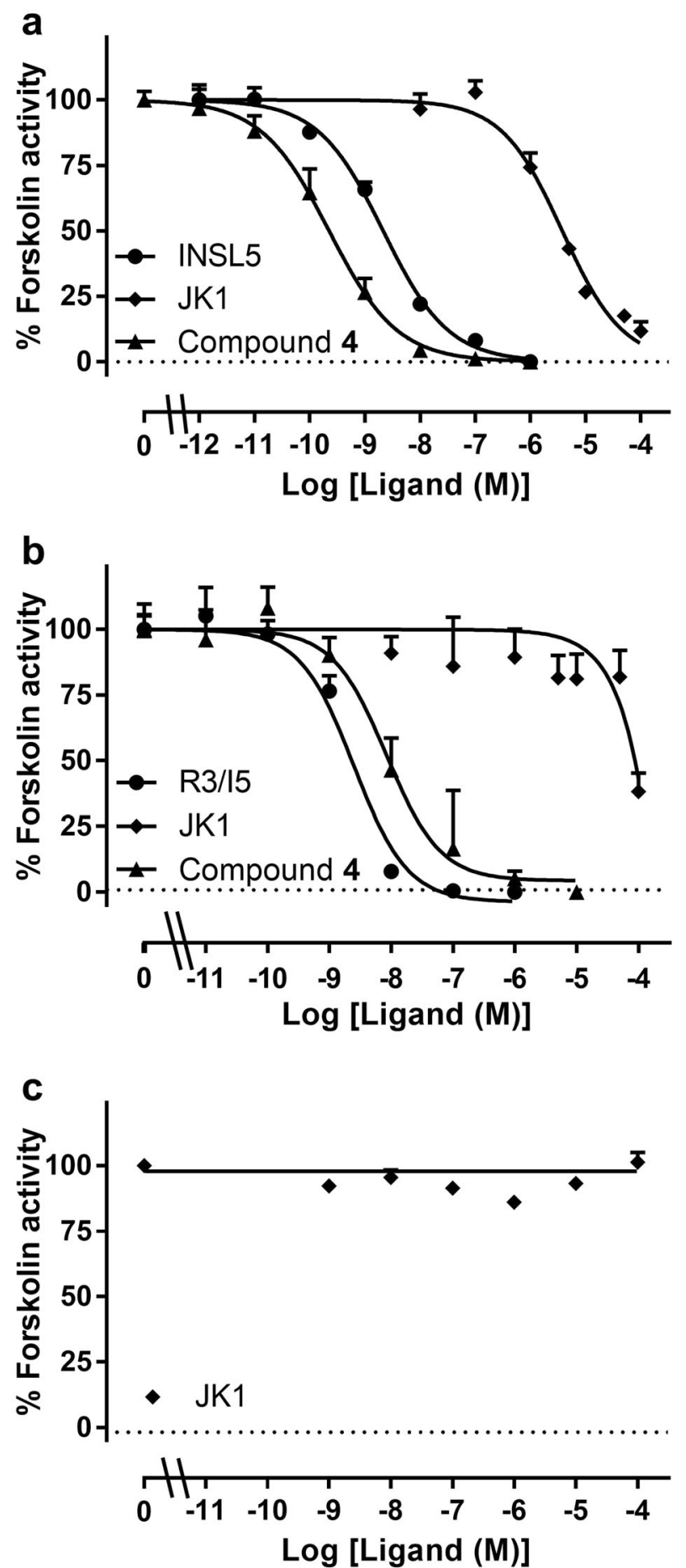

Fig. 5 JK1 activity was confirmed in a pCRE- $\beta$-galactosidase reporter gene assay. a JK1 demonstrated full agonistic activity in CHO-RXFP4 cells. b The compound demonstrated very low activity in $\mathrm{CHO}-$ RXFP3 cells while compound $\mathbf{4}$ showed potent cross-reactivity with RXFP3. c JK1 had no activity in parental CHO cells

In the present study, we developed an HTS assay based on the measurement of intracellular CAMP production, a method that was used in screening modulators of RXFPs $[12,24-26]$. Both human INSL5 and R3/I5 were applied to validate the assay system, and our results (Fig. 1) showed that the assay parameters, such as the $C V, S / B$ ratio and $Z^{\prime}$ factor, were of 

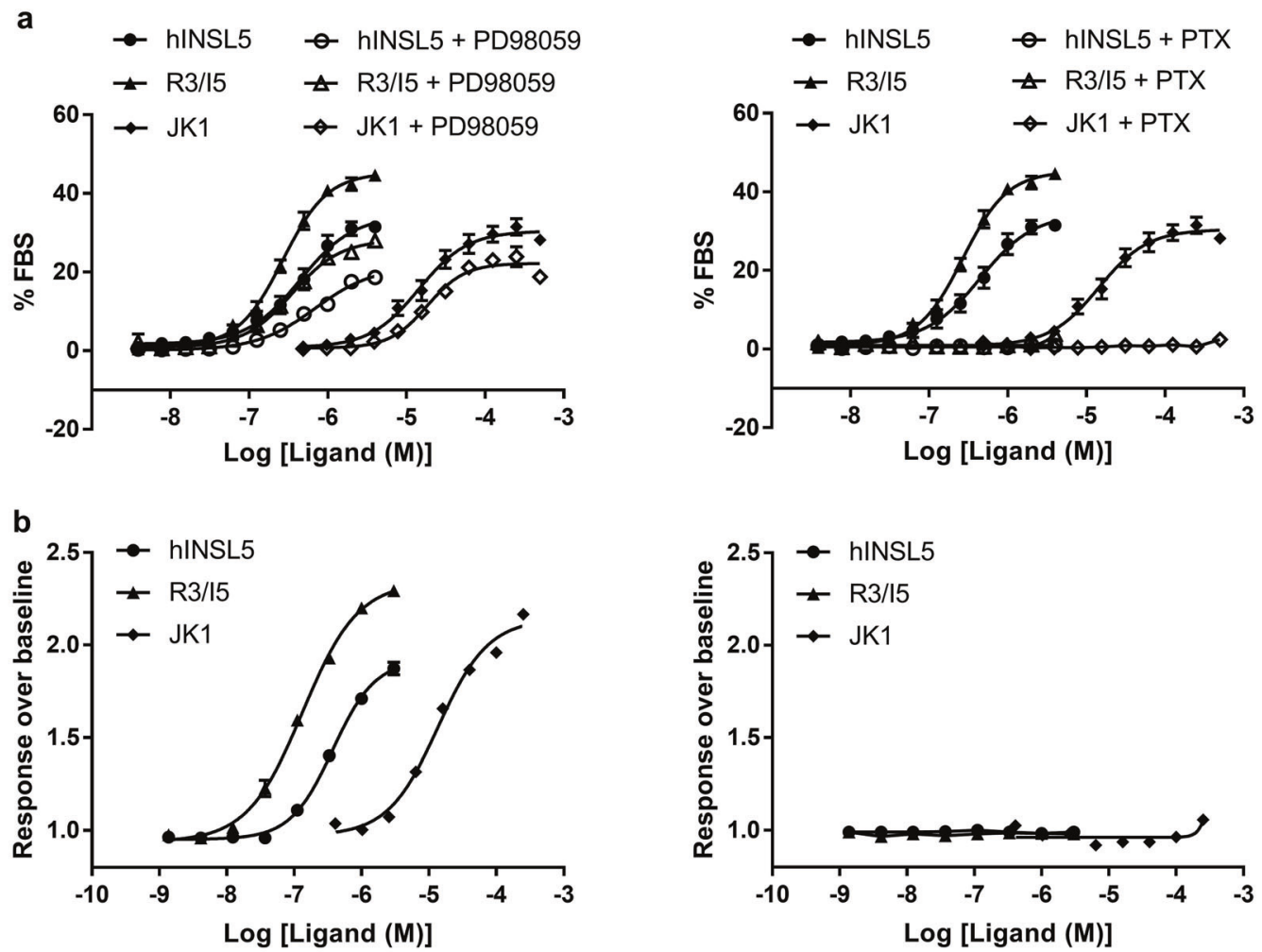

C

RXFP4 interaction with $\beta$-arrestin 1

RXFP4 interaction with $\beta$-arrestin 2

$\rightarrow 1 \mu \mathrm{M}$ hINSL5


Fig. 6 JK1 activates RXFP4-mediated signaling pathways. a JK1 induced receptor-mediated ERK1/2 phosphorylation in hRXFP4-CHO cells which could either be inhibited by PD98059 pretreatment $(20 \mu \mathrm{M}, 2 \mathrm{~h}$, left panel) or be abolished when cells were pretreated with PTX $\left(100 \mathrm{ng} / \mathrm{mL}, 18 \mathrm{~h}\right.$, right panel). b JK1 exhibited a dose-dependent agonistic effect in hRXFP4-G $\alpha_{16}-\mathrm{CHO}$ (left panel) but not $\mathrm{G} \alpha_{16}-\mathrm{CHO}$ cells (right panel). c JK1 weakly promoted RXFP4 interaction with $\beta$-arrestin $1 / 2$

high quality. Most of the 109 initial hits failed in secondary screening or dose-response studies, and only 3 were confirmed, with JK1 (the S-configuration stereoisomer of JK0621-D008) showing a consistent effect on RXFP4-CHO cells (Figs. 2-4). In addition to CAMP, we evaluated the action of JK1 on pCRE activation (Fig. 5), ERK1/2 phosphorylation, intracellular calcium mobilization, and $\beta$-arrestin recruitment (Fig. 6). Activation of all the cell signaling pathways except $\beta$-arrestin recruitment demonstrated that JK1 was a full agonist with similar potency. The poor activity demonstrated in the $\beta$-arrestin recruitment assay may be related to the low potency of the compound and the low sensitivity of this assay. However, since cells naturally expressing RXFP4 are not available, we are unable to verify the results in a nonengineered system at this time. Notably, JK1 also induced toxicity at concentrations close to the $\mathrm{EC}_{50}$ in HEK293T and $\mathrm{CHO}$ cells, which may limit its therapeutic development.
In conclusion, we have established a cAMP-based HTS assay that was used to screen novel RXFP4 agonists. Our HTS campaign and subsequent characterization experiments identified JK1 as a selective agonist for RXFP4. Similar to the endogenous ligand INSL5, JK1 is a full agonist of relevant signaling pathways coupled to RXFP4, including forskolin-stimulated CAMP accumulation, ERK1/2 phosphorylation and $\beta$-arrestin recruitment. Most importantly, JK1 does not activate the closely related RXFP3 receptor. A recent paper [12] outlined a series of small molecule agonists (Table 2) with high affinity for human RXFP4. However, these compounds also demonstrated high affinity for human RXFP3, which we confirmed in this study, and any in vivo studies would therefore be confounded by off-target effects. JK1 represents a novel RXFP4-specific scaffold that could be further developed by medicinal chemistry efforts to study the physiological role of RXFP4. 
Table 2. Summary of identified RXFP4 agonists

\begin{tabular}{|c|c|c|c|c|}
\hline Compound & Structure & Chemical formula & Molecular weight & $\mathrm{EC}_{50}(\mu \mathrm{M})$ \\
\hline
\end{tabular}

Compound 4

\section{ACKNOWLEDGEMENTS}

We are indebted to Ji Wu and Qiang Shen for technical assistance. This work was partially supported by grants from the National Natural Science Foundation of China 81872915 (MWW), 81573479 (DHY), and 81773792 (DHY); the National Science \& Technology Major Project "Key New Drug Creation and Manufacturing Program" of China (2018ZX09735-001 to MWW, 2018ZX09711002-002-005 to DHY and 2018ZX09711002-002-011 to QL); the National Key R\&D Program of China 2018YFA0507000 (MWW); and the Novo Nordisk-CAS Research Fund (NNCAS-20171-CC to DHY). Research at the Florey was supported by the Victorian Government's Operational Infrastructure Support Program. We are grateful to Tania Ferraro and Sharon Layfield for assistance with assays at the Florey. RADB is an NHMRC Senior Research Fellow. The funders had no role in the study design, data collection and analysis, decision to publish, or paper preparation.

\section{AUTHOR CONTRIBUTIONS}

MWW and RADB designed the research. GYL, XQC, ATD, and JL performed the research. YZ established the hRXFP4-Ga ${ }_{16}-\mathrm{CHO}$ cell line. LL and QL synthesized JK1. GYL, LL, QL, DHY, RADB, and MWW analyzed the data. GYL, QL, MWW, and RADB drafted the paper, and GYL, QL, DHY, RADB, and MWW edited and revised the paper.

\section{ADDITIONAL INFORMATION}

The online version of this article (https://doi.org/10.1038/s41401-020-0390-x) contains supplementary material, which is available to authorized users.

Competing interests: The authors declare no competing interests.

\section{REFERENCES}

1. Liu C, Kuei C, Sutton S, Chen J, Bonaventure P, Wu J, et al. INSL5 is a high affinity specific agonist for GPCR142 (GPR100). J Biol Chem. 2005;280:292-300.

2. Fredriksson R, Lagerstrom MC, Lundin LG, Schioth HB. The G-protein-coupled receptors in the human genome form five main families. Phylogenetic analysis, paralogon groups, and fingerprints. Mol Pharmacol. 2003;63:1256-72.
3. Boels K, Schaller HC. Identification and characterisation of GPR100 as a novel human G-protein-coupled bradykinin receptor. $\mathrm{Br} J$ Pharmacol. 2003;140:932-8.

4. Kong RC, Shilling PJ, Lobb DK, Gooley PR, Bathgate RA. Membrane receptors: structure and function of the relaxin family peptide receptors. Mol Cell Endocrinol. 2010;320:1-15.

5. Akhter Hossain M, Bathgate RA, Kong CK, Shabanpoor F, Zhang S, HaugaardJonsson LM, et al. Synthesis, conformation, and activity of human insulin-like peptide 5 (INSL5). ChemBioChem. 2008;9:1816-22.

6. Conklin D, Lofton-Day CE, Haldeman BA, Ching A, Whitmore TE, Lok S, et al. Identification of INSL5, a new member of the insulin superfamily. Genomics. 1999;60:50-6.

7. Ang SY, Hutchinson DS, Patil N, Evans BA, Bathgate RAD, Halls ML, et al. Signal transduction pathways activated by insulin-like peptide 5 at the relaxin family peptide RXFP4 receptor. Br J Pharmacol. 2017;174:1077-89.

8. Luo X, Li T, Zhu Y, Dai Y, Zhao J, Guo ZY, et al. The insulinotrophic effect of insulinlike peptide 5 in vitro and in vivo. Biochem J. 2015;466:467-73.

9. Aparicio S, Dixon J, Hendrick A. Use of GPR100 receptor in diabetes and obesity regulation. US Patent Appl 20080269118.

10. Burnicka-Turek O, Mohamed BA, Shirneshan K, Thanasupawat T, HombachKlonisch S, Klonisch T, et al. INSL5-deficient mice display an alteration in glucose homeostasis and an impaired fertility. Endocrinology. 2012;153:4655-65.

11. Grosse J, Heffron H, Burling K, Akhter Hossain M, Habib AM, Rogers GJ, et al. Insulin-like peptide 5 is an orexigenic gastrointestinal hormone. Proc Natl Acad Sci U S A. 2014;111:11133-8.

12. DeChristopher B, Park SH, Vong L, Bamford D, Cho HH, Duvadie R, et al. Discovery of a small molecule RXFP3/4 agonist that increases food intake in rats upon acute central administration. Bioorg Med Chem Lett. 2019;29:991-4.

13. Luo $X$, Bathgate RA, Zhang WJ, Liu YL, Shao XX, Wade JD, et al. Design and recombinant expression of insulin-like peptide 5 precursors and the preparation of mature human INSL5. Amino Acids. 2010;39:1343-52.

14. Patil NA, Hughes RA, Rosengren KJ, Kocan M, Ang SY, Tailhades J, et al. Engineering of a novel simplified human insulin-like peptide 5 agonist. J Med Chem. 2016;59:2118-25.

15. Belgi A, Hossain MA, Shabanpoor F, Chan L, Zhang S, Bathgate RA, et al. Structure and function relationship of murine insulin-like peptide 5 (INSL5): free C-terminus 
is essential for RXFP4 receptor binding and activation. Biochemistry. 2011;50:8352-61.

16. Van der Westhuizen ET, Sexton PM, Bathgate RA, Summers RJ. Responses of GPCR135 to human gene $3(\mathrm{H} 3)$ relaxin in CHO-K1 cells determined by microphysiometry. Ann N Y Acad Sci. 2005;1041:332-7.

17. Zhang JH, Chung TD, Oldenburg KR. A simple statistical parameter for use in evaluation and validation of high throughput screening assays. J Biomol Screen. 1999;4:67-73.

18. Bathgate RA, Ivell R, Sanborn BM, Sherwood OD, Summers RJ. International union of pharmacology LVII: recommendations for the nomenclature of receptors for relaxin family peptides. Pharmacol Rev. 2006;58:7-31.

19. Faure M, Voyno-Yasenetskaya TA, Bourne HR. CAMP and beta gamma subunits of heterotrimeric $\mathrm{G}$ proteins stimulate the mitogen-activated protein kinase pathway in COS-7 cells. J Biol Chem. 1994;269:7851-4.

20. Crespo $P, X u N$, Simonds WF, Gutkind JS. Ras-dependent activation of MAP kinase pathway mediated by G-protein beta gamma subunits. Nature. 1994:369:418-20.
21. Lopez-llasaca M, Crespo P, Pellici PG, Gutkind JS, Wetzker R. Linkage of G proteincoupled receptors to the MAPK signaling pathway through PI3-kinase gamma. Science. 1997;275:394-7.

22. Bathgate RAD, Halls ML, van der Westhuizen ET, Callander GE, Kocan M, Summers RJ, et al. Relaxin family peptides and their receptors. Physiol Rev. 2013;93:405-80.

23. Hu MJ, Shao XX, Wang JH, Wei D, Guo YQ, Liu YL, et al. Mechanism for insulin-like peptide 5 distinguishing the homologous relaxin family peptide receptor 3 and 4 . Sci Rep. 2016;6:29648.

24. Alvarez-Jaimes L, Sutton SW, Nepomuceno D, Motley ST, Cik M, et al. In vitro pharmacological characterization of RXFP3 allosterism: an example of probe dependency. PLoS ONE. 2012;7:e30792.

25. Chen CZ, Southall N, Xiao J, Marugan JJ, Ferrer M, Hu X, et al. Identification of small-molecule agonists of human relaxin family receptor 1 (RXFP1) by using a homogenous cell-based cAMP assay. J Biomol Screen. 2013;18:670-7.

26. Xiao J, Huang Z, Chen CZ, Agoulnik IU, Southall N, Hu X, et al. Identification and optimization of small-molecule agonists of the human relaxin hormone receptor RXFP1. Nat Commun. 2013;4:1953. 